\title{
Effects of Dog-Assisted Therapy in Adolescents with Eating Disorders: A Study Protocol for a Pilot Controlled Trial
}

\author{
Ana Myriam Lavín-Pérez ${ }^{1}$, Cristina Martín-Sánchez ${ }^{2}$, Beatriz Martínez-Núñez ${ }^{3}$, Luis Lucio Lobato-Rincón ${ }^{2,4}$, \\ Santos Villafaina ${ }^{5, *}$, Israel González-García ${ }^{2}$, Ana Mata-Cantero ${ }^{6}$, Montserrat Graell ${ }^{3,7}$, \\ Eugenio Merellano-Navarro ${ }^{8, *}$ and Daniel Collado-Mateo ${ }^{1,2}$ (D)
}

1 Centre for Sport Studies, Rey Juan Carlos University, Fuenlabrada, 28943 Madrid, Spain; am.lavin.2018@alumnos.urjc.es (A.M.L.-P.); daniel.collado@urjc.es (D.C.-M.)

2 Animal-Assisted Intervention Office, Rey Juan Carlos University, Móstoles, 28933 Madrid, Spain; c.martinsanch@alumnos.urjc.es (C.M.-S.); luislucio.lobato@urjc.es (L.L.L.-R.); oficina.iaa@urjc.es (I.G.-G.)

3 Psychiatry and Clinical Psychology Department, Hospital Infantil Universitario Niño Jesús, 28943 Madrid, Spain; beamartinezn@gmail.com (B.M.-N.); montserratgraell1@gmail.com (M.G.)

4 Department of Psychology, King Juan Carlos University, Alcorcón, 28943 Madrid, Spain

5 Physical Activity and Quality of Life Research Group (AFYCAV), Faculty of Sport Sciences, University of Extremadura, 10003 Caceres, Spain

6 Perroterapia-Animal-Assisted Interventions, Rivas Vaciamadrid, 28943 Madrid, Spain; ana.mata@perroterapia.org

Citation: Lavín-Pérez, A.M.; Martín-Sánchez, C.; Martínez-Núñez, B.; Lobato-Rincón, L.L.; Villafaina, S.; González-García, I.; Mata-Cantero, A.; Graell, M.; Merellano-Navarro, E.; Collado-Mateo, D. Effects of Dog-Assisted Therapy in Adolescents with Eating Disorders: A Study Protocol for a Pilot Controlled Trial. Animals 2021, 11, 2784. https:// doi.org/10.3390/ani11102784

Academic Editor: Benjamin Hart

Received: 2 September 2021

Accepted: 22 September 2021

Published: 24 September 2021

Publisher's Note: MDPI stays neutral with regard to jurisdictional claims in published maps and institutional affiliations.

Copyright: (C) 2021 by the authors Licensee MDPI, Basel, Switzerland. This article is an open access article distributed under the terms and conditions of the Creative Commons Attribution (CC BY) license (https:// creativecommons.org/licenses/by/ $4.0 /)$.
7 Centro de Investigación Biomédica en Red de Salud Mental (CIBERSAM), 28943 Madrid, Spain

8 Grupo de Investigacion EFISAL, Universidad Autónoma de Chile, 3460000 Talca, Chile

* Correspondence: svillafaina@unex.es (S.V.); emerellano@gmail.com (E.M.-N.)

Simple Summary: Animal-assisted therapies may lead to benefits in anxiety, depression, low selfesteem, or quality of life. These variables are commonly present among patients with eating disorders. Thus, the current pilot study will aim to evaluate the effects of a dog-assisted therapy on the eating disorders symptoms, mental, psychosocial, and physical health, quality of life, and handgrip strength of adolescents suffering from eating disorders. Thirty-two patients will participate and 16 of them will receive the intervention while the other 16 will be the control group. Both groups will continue with their treatments prescribed at the hospital, but the experimental group will participate in a dog-assisted therapy program involving 7 sessions in 7 weeks. This will be the first study to examine the effects of dog-assisted therapy in this population. Significant improvements, in the primary and secondary outcomes, may be expected based on the known benefits of AAT on self-esteem, stress, and self-control in different populations. Finally, although the program is focused on the improvement of adolescents' health, animal welfare will be a priority in this study.

Abstract: Background: Eating disorders are characterized by a persistent disturbance that alters food intake and it is often accompanied by anxiety, depression, low self-esteem, or reduced functional capacity and quality of life. Animal-assisted therapies (AAT) have shown benefits in these variables in children and adult populations. Thus, the present pilot study will aim to evaluate the effects of a dogassisted therapy on the eating disorders symptoms, mental, psychosocial, and physical health, quality of life, and handgrip strength of adolescents suffering from eating disorders. Methods: The current pilot study will involve 32 patients, who will be assigned to a control or an experimental group. Intervention will be conducted once a week for seven weeks. Neither the experimental nor the control group will discontinue their usual care. The main outcome measures will be the eating disorder symptoms and the health-related quality of life measured with standardized questionnaires, while the secondary variables will be anxiety, depression, character, behavior, strength, and body mass. Conclusions: This pilot-controlled trial will be the first to evaluate the effects of dog-assisted therapy on the physical and mental health of adolescents with eating disorders. Significant improvements, in the primary and secondary outcomes, may be expected based on the known benefits of AAT on self-esteem, stress, and self-control in different populations. Finally, although the program is focused on the improvement of adolescents' health, animal welfare will be a priority in this study. 
Keywords: animal-assisted therapies; anorexia nervosa; bulimia nervosa; mental health

\section{Introduction}

The DSM-5 [1] classifies the different eating and feeding disorders. They are characterized by a persistent disturbance in eating or eating-directed behavior, which leads to altered food consumption or absorption and causes a deterioration of physical health and psychosocial functioning [1]. The etiology of eating disorders is multifactorial, including both biological (genetic causes or abnormal neurotransmitter systems) and psychological risk factors such as low self-esteem, body dissatisfaction or obsessive thinking [2-4]. Most common eating disorders are anorexia nervosa and bulimia nervosa [1], ranged from $1 \%$ to $4 \%$ of European women [5]. Worldwide, anorexia nervosa and bulimia nervosa had a prevalence of 176.2 per 100,000 people [6]. Among adolescents, the prevalence ranged from $0.3 \%$ to $2.2 \%$ for anorexia nervosa and $0.1 \%$ to $2 \%$ for bulimia nervosa [7]. However, epidemiological studies have been focused on bulimia nervosa and anorexia nervosa, omitting other eating disorders such as binge-eating disorder and other specified feeding or eating disorders $[6,8,9]$. This is relevant, as it is estimated that 17.3 million people suffered from binge-eating disorder and 24.6 million people suffered from other specified feeding or eating disorder [10].

The treatment for eating disorders is challenging due to the large individual patients' differences and thoughts [11]. Thus, it is recommended a multidisciplinary treatment, including family-based, psychological, and pharmacological approaches [4]. Some of the symptoms associated with eating disorders have been previously treated through Animal Assisted Therapy (AAT) in studies conducted with different populations and diseases, such as attention-deficit/hyperactivity disorder, anxiety or depression, to enhance psychological and social factors [12-15]. The International Association of Human-Animal Organizations (IAHAIO) defines AAT as a goal-oriented, planned and structured therapeutic intervention directed and/or delivered by health, education or human service professionals [16].

Previous studies $[15,17]$ showed a significant relationship between AAT sessions and self-esteem improvements and reduced anxiety levels [18-20]. Regarding social skills, Whitely [21] and Duckers [22] have shown that AAT sessions, together with teamwork, could improve social skills and contribute to the development of problem-solving strategies. In the case of interventions focused on improving depression (Francis et al., 1985; Souter and Miller, 2007; Veilleux, 2020), AAT showed a significant reduction of depressive symptoms as patient's rediscovered joy, spontaneity, and healthy leisure capacity through the animal, reducing anhedonia. Moreover, improvements in cooperation and self-control and reduction of the patient's anger, agitation, and aggressiveness may be achieved due to the need to communicate with the animal calmly and non-reactive, promoting emotional skills, impulse regulation, and self-control $[13,23,24]$. A previous systematic review hypothesized that the benefits obtained by AAT in psychological health could be due to the liberation of oxytocin [25]. Nevertheless, the results are contradictory [26]. Future studies are encouraged to examine the mechanisms under the effects of AAT on psychological and physiological domains.

Due to the benefits mentioned above and considering that AAT tried to minimize infection risk $[27,28]$ following the current legislation [29], AAT could be a potentially effective tool to improve adolescents' physical and mental health with eating disorders. This would be relevant, as adolescence is a critical age in the development of self-esteem and self-concept $[30,31]$, in which low values of these variables may be related to the development of eating disorders [32,33]. Thus, the present pilot study will propose a dogassisted therapy program in a population of adolescents with eating disorders to improve the main associated symptoms and consequences of the disorder. The current pilot design has been chosen to explore the feasibility of an AAT intervention in adolescents with eating disorders. Nevertheless, considering the absence of scientific literature in the field and 
the study design of the current pilot study, results would be relevant in the field of eating disorders and AAT.

\section{Objectives and Hypothesis}

The main objective of the current pilot parallel controlled clinical trial is to explore the feasibility of an AAT intervention to improve the eating disorder symptoms, the healthrelated quality of life anxiety, depression, adolescent character and behavior, strength, body mass and treatment satisfaction of adolescents with eating disorders through dog-assisted therapy in contrast to a control group. Secondarily, this study also aimed to provide the results on the effects on anxiety, depression, adolescent character and behavior, strength, body mass and treatment satisfaction. However, it must be noted that, as a pilot study, results must be interpreted with caution.

We hypothesized that adolescents who participate in the AAT (dog-assisted therapy) would improve eating disorder symptoms, quality of life and mood while reducing the anxiety and depressive symptoms and their behavioral problems compared to the control group. Handgrip strength could also be improved as a physical manifestation of overall health status [34]. Furthermore, this test has been conducted in eating disorders due to the importance of strength to avoid sarcopenia [35] as well as to evaluate the physical risk [36]. Besides, the treatment satisfaction is expected to be favorable to patients, families, and the medical staff from the hospital.

\section{Materials and Methods}

\subsection{Study Design}

The current pilot study, a parallel-group non-randomized clinical trial, aimed to analyze the effectiveness of a dog-assisted therapy in adolescents with eating disorders compared to the control group that will receive their usual care. The study setting where the intervention will be carried out is the Niño Jesús University Children's Hospital (Psychiatry and Clinical Psychology Department) in Madrid, Spain. This study protocol followed the SPIRIT 2013 Statement Items to correctly fulfil the standard protocol items for clinical trials [37]. If during the study process any substantial modification is needed, the relevant parties involved will be informed (investigators, hospital's ethics committee, and trial registration platform).

\subsection{Ethical Approval and Trial Registration}

The protocol has been approved by the Ethics Committee of the Niño Jesús University Children's Hospital in March 2021 with the approval number of R-0007/21. After the positive response, the trial was registered at ClinicalTrials.gov under the identification number NCT04869423.

\subsection{Participants}

\subsubsection{Eligibility Criteria}

Participants will need to fulfil the following inclusion criteria in order to be included in the study: (a) adolescents aged $<18$ (b) treated in the Day Hospital of the Psychiatry and Psychology Service of the Niño Jesús Children's University Hospital, (c) to have been diagnosed with any kind of eating disorder (anorexia nervosa, bulimia nervosa, binge eating or other specified feeding or eating disorder) according to the DSM-5 criteria, (d) adolescents willing to participate and available to assist, (e) having read, agreed and signed the written informed consent by patients and their legal guardians. Moreover, patients with dog allergy, dog phobia and history of impulsive animal aggression will be excluded from the study.

\subsubsection{Recruitment}

Initial recruitment, screening, and baseline assessment for the study will be conducted between April and October 2021. The participants' enrollment will be voluntary, and the 
day hospital patients from the Niño Jesús University Children's Hospital will offer them to be part of the study. If they are interested, the detailed information of the study will be explained to their legal guardians so they can approve, by signing the informed consent, the adolescents' participation. Participation in this study is voluntary, and the involvement in the AAT group is conditioned by medical criteria, time period, and the availability to attend the sessions. Thus, the recruitment procedure is resulting in a sample of opportunity.

\subsubsection{Randomization and Blinding}

The current pilot study is a non-randomized pilot-controlled trial. Although participants will know their group assignment, the evaluators will be blinded to the assignment. An anonymous identification code will be assigned to each participant to preserve confidentiality so that the evaluators will not know patients' personal data or recognize the allocation group.

\subsection{Intervention}

The intervention will last nine weeks, including one evaluation session in the first week, seven intervention (or usual care) weeks, and another evaluation session in the last week. Given that there are no previous studies with similar aims, this duration was set as a consensus among all the researchers involved in the study, including the medical staff from the psychiatry unit, the professional handlers, and the academics.

Dogs will follow a strict zoonosis protocol, including behavioral, blood, urine, and feces analyses. Deworming, rabies, and tetravalent vaccinations were also required. Animal welfare will be ensured by the Animal Assisted Intervention Office of the University through a protocol that includes not only the physical health of the animal but also tries to reduce or avoid the presence of fatigue or stress during AAT sessions and also during training. Thus, the handler and the dog must have a strong mutual bond so the handler can rapidly and adequately interpret the dog's body language and identify potential stressful or uncomfortable situations. Any session can be interrupted, and any activity can be skipped if the handler thinks that his/her dog is not having a good time.

\subsubsection{Experimental Group}

The planned intervention will have a length of seven weeks, where patients will participate in a dog-assisted therapy once per week (seven sessions in total). The intervention will be added to the usual care they will be receiving at the Hospital, so they will not have to discontinue any treatment. In order to have complete control of the adolescents and the dogs, the therapy will be carried out in small groups composed of four patients.

Adolescents who will be discharged from the Hospital will have the opportunity to continue with the intervention. If during the intervention any patient presents an allergy or had aggressive behavior toward the dog, the expert will exclude the participant from the experimental group. Besides participating in the dog-assisted therapy program, patients will continue with their usual hospital and/or pharmacological treatments.

As AAT should be directed by health professionals, the sessions will be directed by a psychologist accompanied by two dog handlers. Dog handlers' responsibilities are: (a) to be responsible at all times for the dog's interaction; (b) to identify him/herself and the dog before accessing at the hospital; (c) to ensure dog hygiene and well-being conditions; and (d) to provide necessary material for the development of the activity.

The planned sessions, with a duration of $50 \mathrm{~min}$, will include a welcome part aimed to get in touch with the dog $(5 \mathrm{~min})$, a main part where participants will learn basic notions about dog training and then try to train the dogs (40 $\mathrm{min}$ ) and a closing part to say goodbye to the dogs $(5 \mathrm{~min})$. In the main part, patients will perform different activities and exercises with the dog to progressively work the following objectives to improve patients mental and physical health. The intervention will include the following objectives (two or more different aims can be addressed in the same session): (1) creating a patient-dog bond (2 sessions); (2) decreasing anxiety (7 sessions); (3) improving mood (7 sessions); (4) improve 
social abilities (4 sessions); (5) increasing impulse control (5 sessions); and, (6) enhance self-esteem ( 5 sessions) (See Figure 1$)$.

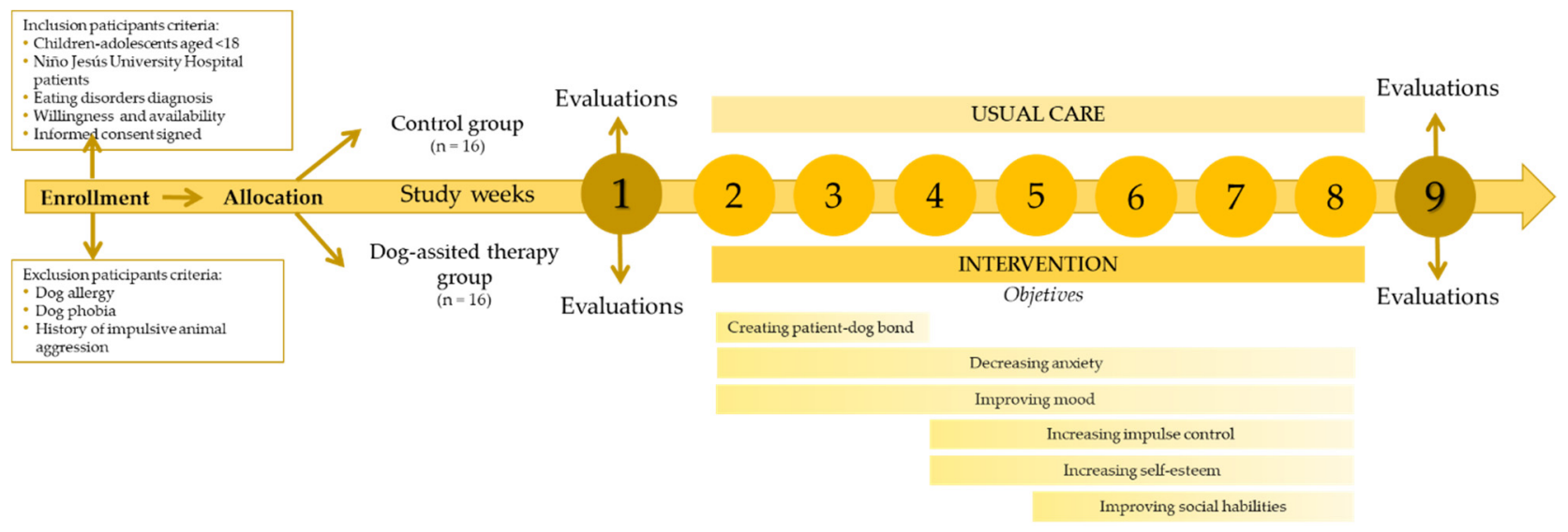

Figure 1. Study timeline and intervention objectives distribution during the dog-assisted therapy sessions.

In this regard, in the first two weeks of the interventions, we could find activities where adolescents will interact with dogs freely, as well as activities where they will have to groom the dogs. Furthermore, in these sessions, adolescents would learn different training techniques and skills that they can use with the dogs, as well as they, will know the importance of therapy dogs in different institutions and patients. These activities will create a patient-dog bond. After this introductory period, in the following five weeks, participants will perform activities of dog training where adolescents, for instance, must choose a behavior to teach the dog between different options, show the training results to the other participants or even play a question-and-answer game where the success depends on the bond between the dogs and the adolescents. The program was designed to maximize the physical contact with the animal (always according and considering the preferences of the participants), as it may be one of the main aspects contributing to the effectiveness of AAI programs [38].

\subsubsection{Control Group}

The control group will have the same inclusion and exclusion criteria as the experimental group. This group will continue with the treatment carried out within the Spanish Public Health System (usual care) provided by the Niño Jesús University Children's Hospital. This usual care may involve pharmacological and non-pharmacological treatments.

\subsection{Measures and Procedures of the Outcome Measures}

All the assessments will be carried out in the Psychiatry and Clinical Psychology Department of the Niño Jesús University Children's Hospital. Participants' sociodemographic information will be collected. Variables such as age, gender, patients' and parents' country, place of residence, or educational level will be asked. Moreover, information regarding disease diagnosis (type of eating disorder and time from diagnosis), pharmacological treatment, and current and/or former animals at home will be collected.

\subsubsection{Eating Disorder Symptoms}

The Spanish version of the Eating Disorder Inventory (EDI-2) [39] will be employed to evaluate the evolution of anorexia nervosa and bulimia nervosa symptoms [40]. The EDI-2 is a 91-item scale with 11 subscales (Drive for Thinness, Bulimia, Body Dissatisfaction, Ineffectiveness, Perfectionism, Interpersonal Distrust, Interoceptive Awareness, Maturity Fears, Asceticism, Impulse Regulation, Social Insecurity). Responses are represented in a Likert scale from 5 (usually) to 0 (never). Higher scores mean more severe symptoms. Thus, total score represents the outcome measure for eating disorder symptoms. 


\subsubsection{Health-Related Quality of Life}

Health-related quality of life will be assessed with the Kidscreen-10 Index [41]. It will allow comparing patients' results to the common population average which provides the limit to classify the results as "normal" (when the values are in the average) or "sensible" (if the scores are below the average). The measure tool consists of 10 items. This index has a high internal consistency (Cronbach's $\alpha$ of 0.82 ) and good retest reliability $(r=0.73$; ICC $=0.72$ ). Scores can range between 10 and 50, with higher score meaning better healthrelated quality of life [41].

\subsubsection{Anxiety}

The State-Trait Anxiety Inventory for children (STAI-C) questionnaire will be used to assess the adolescents' anxiety [42]. The Spanish version, composed of two different scales, one to assess state anxiety ( 20 items) and the other to evaluate trait anxiety ( 20 items) in adolescents [43] will be employed. Each item is rated from 1 to 3 , so the sub-scale scores can range from 20 to 60 . Higher scores are interpreted as higher anxiety [43].

\subsubsection{Depression}

Depression will be measured by the Children Depression Inventory (CDI) [44]. This questionnaire includes 27 items with three possible options from 0 (absence of symptoms) to 2 (severe symptoms). For the current study, the validated Spanish version will be used [45]. The total score ranges from $0-54$, with higher scores being interpreted as higher depression. This scale had a Cronbach's $\alpha$ between 0.75 and 0.94 and a test reliability of 0.84 .

\subsubsection{Adolescent Character}

The Spanish version of the Temperament and Character Inventory-Revised [46] will be employed to assess the patients' character [47]. It consists of 240 items and 5 options for each one. It measures 4 temperaments, Novelty Seeking (NS), Harm Avoidance (HA), Reward Dependence (RD), and Persistence (PS), and three characters, Self-directedness (SD), Cooperativeness (CO), and Self-transcendence (ST). Higher scores mean higher levels in each dimension.

\subsubsection{Adolescent Behavior}

To evaluate the patients' behavior, the Spanish Child Behavior Checklist inside the Achenbach System of Empirically Based Assessment will be used [42,48]. A total of 113 items conformed the scale. In order to get detailed information of participants behaviors, items are grouped in the following 8 subdimensions: anxiety-depression, isolationdepression, somatic complaints, social problems, thinking problems, attentional problems, break the rules conduct, and aggressive behavior. It is scored from $0=$ "not true" to $2=$ "true". Scores range from 0 to 226. Higher scores mean a worse outcome [48].

\subsubsection{Strength}

Patients' strength will be assessed through the isometric maximum strength using a handgrip dynamometer (Takei TKK 5401 Digital Handgrip Dynamometer, Tokyo, Japan). Adolescents will have to press with the hand while it is entirely straight [35]. This test has been employed before in anorexia nervosa patients due to the importance of strength to avoid sarcopenia in this population [35].

\subsubsection{Body Mass Index}

Adolescents' weight and height will be assessed by using a SECA weighing device (SECA, Hamburg, Germany). Body mass index (BMI) will be calculated as follow: BMI $=$ weight $(\mathrm{kg}) \div$ height $^{2}(\mathrm{~m})$. 


\subsubsection{Treatment Satisfaction}

Treatment satisfaction will be evaluated by the treatment satisfaction scale (CRES-4) Feixas i Viaplana, et al. [49]. This questionnaire has three dimensions: satisfaction (general satisfaction with the way the therapist dealt with the problem), problem-solution (how the treatment has helped in relation to the problem) and perception of emotional change (selfperception about the improvements in the emotional state after the treatment). The score in each dimension ranges from 0 to 100 and the global score is the sum of the dimensions (from 0 to 300). Higher scores mean higher satisfaction [49]. Furthermore, a short interview will be answered by participants once the intervention is finished in order to know their opinion (strengths and limitations) of the sessions and the potential benefits.

\subsection{Data Analysis}

All the outcome results will be included in an anonymous database to conduct the statistical analyses. A descriptive, quantitative, and graphics analysis will be performed of all the included variables. The statistic software employed will be the IBM Statistical Package for the Social Sciences (version 25.0; SPSS, Inc., Chicago, IL, USA) [50].

First of all, the Kolmogorov-Smirnov and Shapiro-Wilk tests will be performed to decide the implementation of parametric or non-parametric statistical analyses. Due to the nature of the study (a pilot study) and the low sample size, non-parametric statistical analyses emerged as the most adequate approach.

In this regard, as non-parametric statistics will be performed, the change from baseline for each variable will be calculated and the Mann-Whitney test performed and we will calculate the $r$ as effect size [51]. Therefore, results will include medians, interquartile ranges, the effect size and the statistical significance (obtained from Mann-Whitney $\mathrm{U}$ test) for each primary and secondary outcome. Due to the large number of planned analyses and the low sample size, the alpha level of significance will be adjusted according to the Benjamini-Hochberg procedure to avoid type I error derived from multiple comparisons [52].

Additionally, if all variables were normally distributed, parametric statistics could be also performed and results could be reported as means and standard deviations. Furthermore, the therapy's effects would be assessed using a repeated-measures MANOVA, and the partial eta squared effect size calculated.

\section{Discussion}

The present pilot study will be the first one evaluating the effects a parallel pilotcontrolled trial based on dog-assisted therapy in adolescents with eating disorders. Two groups will compose the study: an AAT group and a control group. The AAT will last seven weeks, and one psychologist and two dog-handlers will direct it. Control group will continue with the usual care provided by the Psychiatry and Clinical Psychology Department of the Niño Jesús University Children's Hospital. We will assess Eating disorder symptoms, Health-related quality of life, Anxiety, Depression, Adolescent character and behavior, Strength, Body mass and treatment satisfaction before and after the intervention.

The future study has been designed as a pilot study where we will assess the feasibility and usefulness of the AAT intervention in adolescents with eating disorders. This study design has been chosen because it is the first time that Niño Jesús University Children's Hospital was involved in an animal-assisted therapy. Therefore, it is a great opportunity for health professional, adolescents and parents to explore the possible strengths and limitations of this type of interventions. Taking into account the strengths and limitations, a future randomized controlled trial will be performed, increasing the sample size and the duration of the therapy. Thus, as a pilot study, the results must be interpreted with caution, and future studies must confirm the findings of this trial.

As commented above, this will be the first study to evaluate the effects of an AAT using therapy dogs in children or adolescents suffering from eating disorders [53], examining physiological, behavioral, social, and physical variables. The measurement of these 
variables will provide a global perspective of the potential benefits of AAT on the health of adolescents with eating disorders. In this regard, previous studies have evaluated the effects of equine-assisted therapy [54] and dolphin therapies [55] in a population comprised of adult women but not in adolescents. Importantly, AAT seems to improve self-esteem in children and adolescents $[15,56]$. Although the exact mechanisms behind self-esteem improvement after AAT are unknown [15], this is relevant as self-esteem improvement is one of the challenges in eating-disorder treatment [57].

We hypothesized that adolescents who participated in the AAT group would enhance the eating disorder symptoms, quality of life and mood while reducing the anxiety and depressive symptoms and their behavioral problems compared to the control group. In this regard, previous studies have reported improvements in health-related quality of life, anxiety, depression or mood after AAT. This is relevant because all these symptoms are present in people with eating disorders [58-60]. Improvements could be due to calming or stress-relieving [61] that the presence of animals could induce, which is based on the biophilia hypothesis [62]. Furthermore, physical contact has been identified as one of the main mechanisms to achieve relevant benefits from AAT, such as stress reduction or the feeling of being connected and not alone [38]. This could be, hypothetically, due to the liberation of oxytocin that previous studies interpreted as a consequence of human-animal interaction $[25,63,64]$. Thus, the activation of the oxytocin system has been proposed to have a key role in the majority of these reported psychological and psychophysiological effects of AAT [25]. However, due to contradictory findings [26], further studies should confirm the results.

Another theoretical mechanism of AAT suggested by Kruger and Serpell [62] is social mediation, which states that the animal may mediate the interaction among patients and between the patient and the therapist. This, along with the known improvements in selfesteem and self-efficacy [18-20], may explain the expected effectiveness of the program in adolescents with eating disorders.

Regarding animal welfare, dogs participating in AAT may be even positively affected in interventions that are carefully designed when comparing their state before and after the session $[65,66]$. Thus, the well-being of dogs will be a priority in the current study. In this regard, different measures will be established, such as feeding the dog before training, limiting the time of training or ensuring rest between sessions. Additionally, the handler and the dog will live together to ensure that the handler adequately identifies and interprets the body language of the dogs during the sessions, reducing the possibility of suffering from stress, which may be experienced during AAT sessions $[67,68]$.

\section{Limitations}

This pilot study has several limitations that must be disclosed. First, the uncertain health conditions in Spain due to COVID-19 defines the maximum number of participants in each group and may change during the conduction of the study. Furthermore, the use of the mask is always mandatory (at least at the beginning of the study), which may affect the comfort and the communication between patients and between the patient and the staff. In addition, the use of face masks is an important limitation due to the influence of human facial expression in the relationship between participants and dogs [69]. In this regard, dogs are highly attentive to human facial expressions and can discriminate different emotions such as happiness [70] or anger [71]. This is important, as dogs can adjust their behavior according to the emotional signals of others [72]. Thus, when the COVID-19 restriction allows, the face mask will be removed.

Another limitation will be the lack of randomization and double-blinding, as participants will know whether they are in the intervention or control group. Additionally, this activity's performance does not imply the cessation of other therapies, treatments, or activities that the patient is receiving. Therefore, it would be impossible to isolate the effect of these therapies in the adolescents completely. Lastly, the relatively small sample size and the large number of variables that we will assess could lead to insufficient statistical power 
to reach the significance level, so future studies must contrast the preliminary findings of this study.

\section{Conclusions}

The current pilot-controlled trial will be the first to evaluate the effects of dog-assisted therapy on the physical and mental health of adolescents suffering from eating disorders. Significant improvements in the main symptoms of participants may be expected based on the known benefits of AAT on self-esteem, stress, and self-control in different populations. Although the intervention is focused on the improvement of adolescents' health, animal welfare will also be adequately ensured.

Author Contributions: Conceptualization, D.C.-M., C.M.-S., B.M.-N., I.G.-G., A.M.-C. and M.G.; methodology, A.M.L.-P., D.C.-M., L.L.L.-R., S.V. and E.M.-N.; software, A.M.L.-P. and D.C.-M.; validation, C.M.-S., B.M.-N., I.G.-G., A.M.-C. and M.G.; formal analysis, A.M.L.-P., D.C.-M., L.L.L.-R., S.V. and E.M.-N.; investigation, A.M.L.-P., D.C.-M., L.L.L.-R., S.V. and E.M.-N.; resources, D.C.-M., C.M.-S., B.M.-N., L.L.L.-R., I.G.-G., A.M.-C. and M.G.; data curation, D.C.-M., C.M.-S., B.M.-N., L.L.L.R., I.G.-G., A.M.-C. and M.G.; writing—original draft preparation, A.M.L.-P., D.C.-M., L.L.L.-R., S.V. and E.M.-N.; writing-review and editing, D.C.-M., C.M.-S., B.M.-N., L.L.L.-R., I.G.-G., A.M.-C. and M.G.; visualization, D.C.-M.; supervision, E.M.-N., S.V. and I.G.-G.; project administration, D.C.-M., I.G.-G. and M.G.; funding acquisition, D.C.-M., I.G.-G. and M.G. All authors have read and agreed to the published version of the manuscript.

Funding: The author A.M.L.-P. is supported by the Industrial Doctorate Spanish National grant program, part of the Strategic Plan on Science and Innovation Support of the Spanish Ministry of Science, Innovation, and Universities. The predoctoral industry grant identification number is DIN2018-010129.

Institutional Review Board Statement: The study was conducted according to the guidelines of the Declaration of Helsinki, and approved by the Ethics Committee of Niño Jesús University Children's Hospital (protocol code R-0007/21 and March 2021).

Informed Consent Statement: Not applicable.

Acknowledgments: The current study was funded by Purina España (Reg. Trademark of Nestle S.A.) and Rey Juan Carlos University through the Animal Assisted Intervention Office. The funders had no role in study design, data collection, and analysis, decision to publish, or preparation of the manuscript. The authors want to thank Manuel Fonseca García, from Perroterapia, for his labor in the design of the intervention.

Conflicts of Interest: The authors declare no conflict of interest.

\section{References}

1. APA. Diagnostic and Statistical Manual of Mental Disorders (DSM-5®); American Psychiatric Publishing: Washington, DC, USA, 2013.

2. Polivy, J.; Herman, C.P. Causes of eating disorders. Annu. Rev. Psychol. 2002, 53, 187-213. [CrossRef]

3. Gorwood, P.; Duriez, P.; Lengvenyte, A.; Guillaume, S.; Criquillion, S. Clinical insight in anorexia nervosa: Associated and predictive factors. Psychiatry Res. 2019, 281, 112561. [CrossRef] [PubMed]

4. Harrington, B.C.; Jimerson, M.; Haxton, C.; Jimerson, D.C. Initial evaluation, diagnosis, and treatment of anorexia nervosa and bulimia nervosa. Am. Fam. Physician 2015, 91, 46-52. [PubMed]

5. Keski-Rahkonen, A.; Mustelin, L. Epidemiology of eating disorders in Europe: Prevalence, incidence, comorbidity, course, consequences, and risk factors. Curr. Opin. Psychiatry 2016, 29, 340-345. [CrossRef] [PubMed]

6. GBD. Global burden of 369 diseases and injuries in 204 countries and territories, 1990-2019: A systematic analysis for the Global Burden of Disease Study 2019. Lancet (Lond. Engl.) 2020, 396, 1204-1222. [CrossRef]

7. Portela de Santana, M.L.; Ribeiro, H.; Mora Giral, M.; Raich, R.M. La epidemiología y los factores de riesgo de los trastornos alimentarios en la adolescencia: Una revisión. Nutr. Hosp. 2012, 27, 391-401. [PubMed]

8. Hart, L.M.; Mitchison, D.; Hay, P.J. The case for a national survey of eating disorders in Australia. J. Eat. Disord. 2018, 6, 30. [CrossRef] [PubMed]

9. Jacobi, F.; Wittchen, H.U.; Hölting, C.; Höfler, M.; Pfister, H.; Müller, N.; Lieb, R. Prevalence, co-morbidity and correlates of mental disorders in the general population: Results from the German Health Interview and Examination Survey (GHS). Psychol. Med. 2004, 34, 597-611. [CrossRef] 
10. Santomauro, D.F.; Melen, S.; Mitchison, D.; Vos, T.; Whiteford, H.; Ferrari, A.J. The hidden burden of eating disorders: An extension of estimates from the Global Burden of Disease Study 2019. Lancet Psychiatry 2021, 8, 320-328. [CrossRef]

11. Williams, R.; Smith, M.; Wright, D. Anorexia: A literature review of young people's experiences of hospital treatment. Nurs. Child. Young People 2021, 33, 10-17.

12. Koukourikos, K.; Georgopoulou, A.; Kourkouta, L.; Tsaloglidou, A. Benefits of animal assisted therapy in mental health. Int. J. Caring Sci. 2019, 12, 1898.

13. Jones, M.G.; Rice, S.M.; Cotton, S.M. Incorporating animal-assisted therapy in mental health treatments for adolescents: A systematic review of canine assisted psychotherapy. PLoS ONE 2019, 14, e0210761. [CrossRef] [PubMed]

14. Wilson, K.; Buultjens, M.; Monfries, M.; Karimi, L. Equine-Assisted Psychotherapy for adolescents experiencing depression and/or anxiety: A therapist's perspective. Clin. Child. Psychol. Psychiatry 2017, 22, 16-33. [CrossRef] [PubMed]

15. Schuck, S.E.; Johnson, H.L.; Abdullah, M.M.; Stehli, A.; Fine, A.H.; Lakes, K.D. The role of animal assisted intervention on improving self-esteem in children with attention deficit/hyperactivity disorder. Front. Pediatrics 2018, 6, 300. [CrossRef] [PubMed]

16. Jegatheesan, B.; Beetz, A.; Ormerod, E.; Johnson, R.; Fine, A.; Yamazaki, K.; Dudzik, C.; Garcia, R.; Winkle, M.; Choi, G. IAHAIO Whitepaper 2014 (Updated for 2018). The IAHAIO Definitions for Animal Assisted Intervention and Guidelines for Wellnes of Animals Involved in AAI. Available online: http:/ / iahaio.org/best-practice/white-paper-on-animal-assisted-interventions / (accessed on 11 April 2018).

17. Cumella, E.J.; Simpson, S. Efficacy of equine therapy: Mounting evidence. Wickenburg AZ Remuda Ranch Cent. Anorex. Bulimia. 2007, 1, 2013.

18. Barker, S.B.; Dawson, K.S. The effects of animal-assisted therapy on anxiety ratings of hospitalized psychiatric patients. Psychiatr. Serv. 1998, 49, 797-801. [CrossRef]

19. Cruz-Fierro, N.; Vanegas-Farfano, M.; González-Ramírez, M.T. Dog-Assisted Therapy and Dental Anxiety: A Pilot Study. Animals 2019, 9, 512. [CrossRef]

20. Villafaina-Domínguez, B.; Collado-Mateo, D.; Merellano-Navarro, E.; Villafaina, S. Effects of dog-based animal-assisted interventions in prison population: A systematic review. Animals 2020, 10, 2129. [CrossRef]

21. Whitely, R. Therapeutic Benefits of Equine Assisted Psychotherapy for At-Risk Adolescents; Texas A \& M University-Commerce: Commerce, TX, USA, 2009.

22. Duckers, P. The added value of equine-assisted therapy in residential youth care. MACESS Maastricht Neth. $2008,6,1$.

23. Mallon, G.P. Utilization of animals as therapeutic adjuncts with children and youth: A review of the literature. Child Youth Care Forum 1992, 21, 53-67. [CrossRef]

24. McCormick, A.; McCormick, M. Horse Sense and the Human Heart: What Horses can Teach Us about Trust, Bonding, Creativity and Spirituality; Health Communications. Inc.: Deerfield Beach, FL, USA, 1997.

25. Beetz, A.; Uvnäs-Moberg, K.; Julius, H.; Kotrschal, K. Psychosocial and psychophysiological effects of human-animal interactions: The possible role of oxytocin. Front. Psychol. 2012, 3, 234. [CrossRef]

26. Menna, L.F.; Santaniello, A.; Amato, A.; Ceparano, G.; Di Maggio, A.; Sansone, M.; Formisano, P.; Cimmino, I.; Perruolo, G.; Fioretti, A. Changes of Oxytocin and Serotonin Values in Dialysis Patients after Animal Assisted Activities (AAAs) with a Dog-A Preliminary Study. Animals 2019, 9, 526.

27. Creagan, E.T.; Bauer, B.A.; Thomley, B.S.; Borg, J.M. Animal-assisted therapy at Mayo Clinic: The time is now. Complement. Ther Clin. Pract. 2015, 21, 101-104. [CrossRef] [PubMed]

28. Snipelisky, D.; Burton, M.C. Canine-assisted therapy in the inpatient setting. South. Med. J. 2014, 107, 265-273. [CrossRef]

29. DiSalvo, H.; Haiduven, D.; Johnson, N.; Reyes, V.V.; Hench, C.P.; Shaw, R.; Stevens, D.A. Who let the dogs out? Infection control did: Utility of dogs in health care settings and infection control aspects. Am. J. Infect. Control. 2006, 34, 301-307. [CrossRef] [PubMed]

30. Sebastian, C.; Burnett, S.; Blakemore, S.J. Development of the self-concept during adolescence. Trends Cogn. Sci. 2008, 12, 441-446. [CrossRef] [PubMed]

31. Orth, U.; Erol, R.Y.; Luciano, E.C. Development of self-esteem from age 4 to 94 years: A meta-analysis of longitudinal studies. Psychol. Bull. 2018, 144, 1045. [CrossRef]

32. Mora, F.; Rojo, S.F.; Banzo, C.; Quintero, J. The impact of self-esteem on eating disorders. Eur. Psychiatry 2017, 41, S558. [CrossRef]

33. Jacobi, C.; Paul, T.; de Zwaan, M.; Nutzinger, D.O.; Dahme, B. Specificity of self-concept disturbances in eating disorders. Int. J. Eat. Disord. 2004, 35, 204-210. [CrossRef]

34. Soysal, P.; Hurst, C.; Demurtas, J.; Firth, J.; Howden, R.; Yang, L.; Tully, M.A.; Koyanagi, A.; Ilie, P.C.; López-Sánchez, G.F. Handgrip strength and health outcomes: Umbrella review of systematic reviews with meta-analyses of observational studies. $J$. Sport Health Sci. 2021, 10, 290-295. [CrossRef]

35. Etemadi, S.; Sun, G.X.; Leung, S.P.; Siddique, A.; Cooper, S.; Ezenwa, N.C.; Treasure, J. The Sit up Squat Stand test and Hand Grip Strength: What is the role of tests of muscle power in risk assessment in Anorexia Nervosa? Eur. Eat. Disord. Rev. 2021, 29, 670-679. [CrossRef]

36. García-Hermoso, A.; Cavero-Redondo, I.; Ramírez-Vélez, R.; Ruiz, J.R.; Ortega, F.B.; Lee, D.C.; Martínez-Vizcaíno, V. Muscular strength as a predictor of all-cause mortality in an apparently healthy population: A systematic review and meta-analysis of data from approximately 2 million men and women. Arch. Phys. Med. Rehabil. 2018, 99, 2100-2113. [CrossRef] 
37. Chan, A.-W.; Tetzlaff, J.M.; Altman, D.G.; Laupacis, A.; Gøtzsche, P.C.; Krleža-Jerić, K.; Hróbjartsson, A.; Mann, H.; Dickersin, K.; Berlin, J.A. SPIRIT 2013 statement: Defining standard protocol items for clinical trials. Ann. Intern. Med. 2013, 158, 200-207. [CrossRef] [PubMed]

38. Shen, R.Z.Z.; Xiong, P.; Chou, U.I.; Hall, B.J. “We need them as much as they need us": A systematic review of the qualitative evidence for possible mechanisms of effectiveness of animal-assisted intervention (AAI). Complement. Ther. Med. 2018, 41, 203-207. [CrossRef]

39. Urzúa, A.; Castro, S.; Lillo, A.; Leal, C. Evaluación de los trastornos alimentarios: Propiedades psicométricas del test EDI-2 en adolescentes escolarizados (as) de 13 a 18 años. Rev. Chil. Nutr. 2009, 36, 1063-1073. [CrossRef]

40. Garner, D.M.; Olmstead, M.P.; Polivy, J. Development and validation of a multidimensional eating disorder inventory for anorexia nervosa and bulimia. Int. J. Eat. Disord. 1983, 2, 15-34. [CrossRef]

41. Ravens-Sieberer, U.; Erhart, M.; Rajmil, L.; Herdman, M.; Auquier, P.; Bruil, J.; Power, M.; Duer, W.; Abel, T.; Czemy, L. Reliability, construct and criterion validity of the KIDSCREEN-10 score: A short measure for children and adolescents' well-being and health-related quality of life. Qual. Life Res. 2010, 19, 1487-1500. [CrossRef]

42. Seligman, L.D.; Ollendick, T.H.; Langley, A.K.; Baldacci, H.B. The utility of measures of child and adolescent anxiety: A metaanalytic review of the Revised Children's Manifest Anxiety Scale, the State-Trait Anxiety Inventory for Children, and the Child Behavior Checklist. J. Clin. Child. Adolesc. Psychol. 2004, 33, 557-565. [CrossRef]

43. Seisdedos, N. STAIC, Cuestionario de Autoevaluación; TEA Ediciones SA: Madrid, Spain, 1990.

44. Kovacs, M. Children's Depression Inventory (CDI); Multi-Health System Toronto: Toronto, ON, Canada, 2003.

45. Del Barrio, V.; Carrasco, M. CDI Inventario de Depresión Infantil; TEA Ediciones: Madrid, Spain, 2004.

46. Gutiérrez-Zotes, J.; Bayón, C.; Montserrat, C.; Valero, J.; Labad, A.; Cloninger, C.; Fernandez-Aranda, F. Temperament and Character Inventory-Revised (TCI-R). Standardization and normative data in a general population sample. Actas Esp. Psiquiatr. 2004, 32, 8-15.

47. Cloninger, C.R. The Temperament and Character Inventory-Revised; Center for Psychobiology of Personality, Washington University: St. Louis, MO, USA, 1999.

48. Sardinero García, E.; Pedreira Massa, J.; Muñiz, J. El cuestionario CBCL de Achenbach: Adaptación española y aplicaciones clínico-epidemiológicas. Clin. Y Salud 1997, 8, 447.

49. Feixas i Viaplana, G.; Pucurull, O.; Roca, C.; Paz, C.; García Grau, E.; Bados López, A. Escala de Satisfacción con el tratamiento recibido (CRES-4): La versión en español. Rev. Psicoter. 2012, 23, 51-58. [CrossRef]

50. Frey, F. SPSS (software). Int. Encycl. Commun. Res. Methods 2017, 1-2. [CrossRef]

51. Fritz, C.O.; Morris, P.E.; Richler, J.J. Effect Size Estimates: Current Use, Calculations, and Interpretation. J. Exp. Psychol. Gen. 2012, 141, 2-18. [CrossRef] [PubMed]

52. Benjamini, Y.; Hochberg, Y. Controlling the false discovery rate: A practical and powerful approach to multiple testing. J. R. Stat. Soc. Ser. B Methodol. 1995, 57, 289-300. [CrossRef]

53. Stefanini, M.C.; Martino, A.; Allori, P.; Galeotti, F.; Tani, F. The use of Animal-Assisted Therapy in adolescents with acute mental disorders: A randomized controlled study. Complement. Ther. Clin. Pract. 2015, 21, 42-46. [CrossRef]

54. Cumella, E.; Lutter, C.B.; Smith-Osborne, A.; Kally, Z. Equine Therapy in the Treatment of Female Eating Disorder. SOP Trans. Psychol. 2014, 1, 13-21. [CrossRef]

55. Schenk, R.; Schandry, R.; Schenk, S. Animal-Assisted Therapy with Dolphins in Eating Disorders; University Research Collection, Ludwig-Maximilans-University: Munich, Germany, 2009.

56. Hauge, H.; Kvalem, I.L.; Enders-Slegers, M.-J.; Berget, B.; Braastad, B.O. Persistence during tasks with horses in relation to social support, general self-efficacy and self-esteem in adolescents. Anthrozoös 2015, 28, 333-347. [CrossRef]

57. Colmsee, I.-S.O.; Hank, P.; Bošnjak, M. Low Self-Esteem as a Risk Factor for Eating Disorders. Z. Psychol. $2021,229,48-69$.

58. DeJong, H.; Oldershaw, A.; Sternheim, L.; Samarawickrema, N.; Kenyon, M.D.; Broadbent, H.; Lavender, A.; Startup, H.; Treasure, J.; Schmidt, U. Quality of life in anorexia nervosa, bulimia nervosa and eating disorder not-otherwise-specified. J. Eat. Disord. 2013, 1, 43. [CrossRef]

59. Rosenbaum, D.L.; White, K.S. The role of anxiety in binge eating behavior: A critical examination of theory and empirical literature. Health Psychol. Res. 2013, 1, e19. [CrossRef]

60. Singleton, C.; Kenny, T.E.; Hallett, D.; Carter, J.C. Depression partially mediates the association between binge eating disorder and health-related quality of life. Front. Psychol. 2019, 10, 209. [CrossRef] [PubMed]

61. Hunt, M.G.; Chizkov, R.R. Are therapy dogs like Xanax? Does animal-assisted therapy impact processes relevant to cognitive behavioral psychotherapy? Anthrozoös 2014, 27, 457-469. [CrossRef]

62. Kruger, K.A.; Serpell, J.A. 3-Animal-assisted interventions in mental health: Definitions and theoretical foundations. In Handbook on Animal-Assisted Therapy, 3rd ed.; Fine, A.H., Ed.; Academic Press: San Diego, CA, USA, 2010; pp. 33-48. [CrossRef]

63. Nagasawa, M.; Kikusui, T.; Onaka, T.; Ohta, M. Dog's gaze at its owner increases owner's urinary oxytocin during social interaction. Horm. Behav. 2009, 55, 434-441. [CrossRef] [PubMed]

64. Julius, H.; Beetz, A.; Kotrschal, K.; Turner, D.; Uvnäs-Moberg, K. Attachment to Pets: An Integrative View of Human-Animal Relationships with Implications for Therapeutic Practice; Hogrefe Publishing: Göttingen, Germany, 2012. 
65. Clark, S.D.; Martin, F.; McGowan, R.T.; Smidt, J.M.; Anderson, R.; Wang, L.; Turpin, T.; Langenfeld-McCoy, N.; Bauer, B.A.; Mohabbat, A.B. Physiological state of therapy dogs during animal-assisted activities in an outpatient setting. Animals 2020, 10, 819. [CrossRef] [PubMed]

66. Clark, S.D.; Smidt, J.M.; Bauer, B.A. Welfare considerations: Salivary cortisol concentrations on frequency of therapy dog visits in an outpatient hospital setting: A pilot study. J. Vet. Behav. 2019, 30, 88-91. [CrossRef]

67. Haubenhofer, D.K.; Kirchengast, S. Physiological arousal for companion dogs working with their owners in animal-assisted activities and animal-assisted therapy. J. Appl. Anim. Welf. Sci. 2006, 9, 165-172. [CrossRef]

68. Haubenhofer, D.K.; Kirchengast, S. 'Dog handlers' and dogs' emotional and cortisol secretion responses associated with animalaassisted therapy sessions. Soc. Anim. 2007, 15, 127-150. [CrossRef]

69. Correia-Caeiro, C.; Guo, K.; Mills, D.S. Perception of dynamic facial expressions of emotion between dogs and humans. Anim. Cogn. 2020, 23, 465-476. [CrossRef]

70. Nagasawa, M.; Murai, K.; Mogi, K.; Kikusui, T. Dogs can discriminate human smiling faces from blank expressions. Anim. Cogn. 2011, 14, 525-533. [CrossRef]

71. Albuquerque, N.; Guo, K.; Wilkinson, A.; Savalli, C.; Otta, E.; Mills, D. Dogs recognize dog and human emotions. Biol. Lett. 2016, 12, 20150883. [CrossRef]

72. Proops, L.; Grounds, K.; Smith, A.V.; McComb, K. Animals remember previous facial expressions that specific humans have exhibited. Curr. Biol. 2018, 28, 1428-1432. [CrossRef] [PubMed] 